\title{
Recent Advances in Small-Scale Mechanical Property Measurement by Nanoindentation
}

\author{
George M. Pharr \\ University of Tennessee, Knoxville, TN 37996-2100; and \\ Oak Ridge National Laboratory, Oak Ridge, TN 37830
}

Since its initial development in the early 1980's [1], nanoindentation has matured into one of the premier testing techniques for measuring mechanical properties at the micrometer and submicrometer scales and has emerged as a critical tool that has helped to shape the nanotechnology revolution. At the heart of the technique are testing systems with simple but precise force actuators and displacement measuring devices that record the force-displacement record as a diamond indenter, usually the form of a pyramid or a sphere, is pressed into and withdrawn from a small region in the surface of a material of interest. The nano-scale force-displacement data, which can be obtained with a spatial resolution as small as a few nanometers, contains a wealth of information about the local mechanical properties [2-4]. This enables the mechanical characterization of very thin films, like those used in the semiconductor, magnetic storage, and hard coatings industries, as well as very small precipitates, particles and second phases, many of which may not exist in bulk form and cannot be characterized by traditional mechanical testing methods. Computer automation of nanoindentation testing systems now routinely provides for complete two-dimensional mapping of properties over regions stretching from sub-micron to millimeters in scale.

In the early days of nanoindentation research, the focus was primarily on the measurement of two fundamental mechanical properties: the hardness, $H$, a simple but important measure of the material's resistance to plastic deformation, and the elastic modulus, $E$, the most fundamental measure of a material's elastic behavior. There was also intense activity in establishing how substrates influence the measurements so that the properties of very thin films could be unambiguously determined independent of their substrates. In the first two decades of nanoindentation research, methods for making these measurements were fully developed and/or the limitations of exactly what could be measured were established. Since then, the focus has shifted to a variety of other properties that can be measured at the sub-micron and nano-scales such as creep, viscoelasticity, and fracture, and improving systems to allow for measurements at extremes of temperature and in a variety of environments such as liquids, gases, and vacuum. The articles in this issue review important recent developments in these areas.

In the first article, Wheeler et al. [5] provide an up-to-date overview of advances made in nanoindentation testing at elevated temperatures. Such measurements are fraught with difficulties, not the least of which is the thermal drift caused by local thermal transients that produce expansion or contraction of parts of the testing system that add to the displacements measured by the system and which are notoriously difficult to separate out from the real displacements in the sample. Progress towards minimizing these effects has been made mostly through the realization that both the sample and the indenter tip must both be heated to precisely the same temperature, with methods for achieving this and the technical difficulties discussed in detail in the article. The authors also discuss the importance of identifying alternatives to diamond for use as hard and stiff materials for indenters. Diamond has limitations in high temperature testing due to its chemical stability in oxidizing environments and its potential for 
chemical reactivity with the indented material. For example, the authors show how rapidly diamond indenters can degrade when indenting steel at $600{ }^{\circ} \mathrm{C}$.

The article by Durst and Maier [6] further addresses fundamental issues of high temperature testing by examining how parameters characteristic of creep and the thermally activated processes that control elevated temperature plasticity can be measured by nanoindentation methods. The methods they explore are the indentation equivalents of constant load creep tests, stress relaxation tests, and strain rate change tests. Once again, thermal drift is a significant issue, but the authors demonstrate how its effects can be at least partially mitigated by employing dynamic stiffness measurement techniques in conjunction with a precise knowledge of the elastic constants of the material at the test temperature. They also discuss the non-trivial issue of how indentation creep data can be used to derive conventional creep parameters like those measured in uniaxial tension or compression tests, since these are what are most often needed for engineering design purposes. These relationships are not at all straightforward because of the non-linearity of the plastic deformation processes coupled with the non-uniform nature of the stresses in the material around the indenter. The relationships are also complicated by transient behavior, which is more complex for non-uniform indentation stress fields, but can potentially be ignored once a steady state sets in, provided the steady state condition can be adequately identified. The authors also document other complications in deriving macroscopic creep parameters from nanoindentation measurements that arise from size effects unique to small-scale testing. In particular, when mechanical properties are measured at small scales, materials often behave as if they were stronger, giving rise to the adage "smaller is stronger".

The measurement of time dependent mechanical properties is also the primary subject of interest in the article Herbert et al. [7], which details new nanoindentation methods for assessing the viscoelastic and viscoplastic properties of polymeric materials. These developments are driven by an ever-increasing need for small-scale measurements in the soft materials used in a wide variety of applications such as thin polymer coatings, polymer fuel cell membranes, biological tissues, and materials for nano-imprinting. The properties of interest include the storage and loss modulus, creep compliance, and stress relaxation modulus, all of which can be measured either in the time domain through indentation-based creep or stress relaxation experiments or in the frequency domain by cyclic indentation with phase-lock amplifiers. All these measurement techniques share one feature in common, specifically, that accurate measurement of properties can be achieved only if specimen-machine interactions are carefully modeled and analyzed. The authors also discuss the importance of accurately identifying the point of surface contact, which is much more difficult in soft polymeric materials than in hard metals and ceramics, as well as complications arising from thermal drift and from the permanent plastic deformation that can occur during indentation. Although methods for reducing the errors are succinctly outlined, the authors are careful to note that none of them is foolproof, and there is still need for further development in this area. The article concludes by noting the pressing need for standard reference materials that can be used to calibrate machine dynamics and assess the accuracy of new test methodologies.

One entirely new area in which nanoindentation techniques are being rapidly adopted is the mechanics of soft biological tissues, and the article by Oyen [8] details the challenges in measuring these materials. The discussion focuses mostly on the mechanical behavior of 
hydrated materials and gels, to include biological tissues and hydrogels, in which the viscous movement of unbound water through the structure can play an important role in establishing the time-dependent mechanical behavior. Such materials are often modeled as poro-elastic or poroviscoelastic, with an associated time dependence that can potentially be characterized at small scales by nanoindentation testing. However, accurately making such measurements requires special new techniques such as testing in wet conditions, careful consideration of liquid capillarity on the measured forces, and very sensitive methods for surface detection in what are among the most compliant of all materials. There are also size effects related to the dimension of the indentation contact relative to the average pore size. Collectively, all these effects add to the observed complexity in nanoindentation force-displacement behavior and lead to the conclusion that much remains to be done before we can accurately model and extract basic poro-elastic and poro-viscoelastic parameters using nanoindentation methods.

The final article deals with an entirely different subject, the measurement of fracture toughness at small scales by nanoindentation. If successfully developed, such methods could become especially valuable in the development new hard coatings used to improve the durability and wear resistance of many advanced engineering materials. After briefly reviewing the stateof-the-art, Sebastiani et al. [9] describe a new technique based on the splitting of cylindrical pillars prepared by focused ion beam (FIB) milling using nanoindentation with pyramidal indenters. The basic idea is that as radial cracks form and grow from the corners of the contact impression during the indentation of a pillar, a mechanical instability is reached at which the cracks catastrophically propagate to the outer free surface at the circumference of the pillar. This produces a very reproducible displacement burst in the indentation force-displacement data, which, in conjunction with finite element analysis, can be used to measure the fracture toughness without the need for imaging the crack lengths, as is usually required by the other techniques. The new method has the added advantage that FIB milling the pillar removes any in-plane residual stresses, which complicate and produce uncertainties in data interpretation for many of the other techniques. When applied to several materials with independently measured fracture toughnesses, the new technique appears quite promising.

In closing, we are indebted to the authors for providing excellent manuscripts that describe activity at the cutting edge of a field that is rapidly expanding into numerous new disciplines and crisscrossing the worlds of soft and hard materials. Their contributions clearly demonstrate that even after three decades of relatively intense development, nanoindentation research remains a healthy and vibrant research area with many new horizons to explore.

\section{Acknowledgements}

Support provided the U.S. Department of Energy, Office of Basic Energy Sciences, Materials Sciences and Engineering Division, and by the National Science Foundation under grant DMR 1427812.

\section{$\underline{\text { References }}$}

[1] Pethica JB, Hutchings R, and Oliver WC, Hardness measurement at penetration depths as small as $20 \mathrm{~nm}$. Philos. Mag. 48 593-606 (1983). 
[2] Doerner MF and Nix WD, A method for interpreting the data from depth-sensing indentation instruments J. Mater. Res. 1, 601-09 (1986).

[3] Oliver WC and Pharr GM, An improved technique for determining hardness and elastic modulus using load and displacement sensing indentation experiments, J. Mater. Res. 7 1564-83 (1992).

[4] Oliver WC and Pharr GM, Measurement of hardness and elastic modulus by instrumented indentation: Advances in understanding and refinements to methodology, J. Mater. Res. 19 320 (2004).

[5] Wheeler JM, Armstrong D, Heinz W, and Schwaiger R, this volume.

[6] Durst K and Maier V, this volume.

[7] Herbert EG, Phani PS, and Johanns KE, this volume.

[8] Oyen ML, this volume.

[9] Sebastiani M, Johanns KE, Herbert EG, and Pharr GM, this volume. 